\section{Violence: from knowledge to prevention}

\author{
Pamela Orpinas and \\ Rebecca de los Ríos, \\ guest editors
}

Violence-in its various forms and manifestations-is undoubtedly one of the issues of greatest importance in the Americas and of highest concern to governments, civil society, and international organizations. Violence is affected by multiple political, economic, and cultural factors that have irreparable consequences for individuals, families, and different population groups. Violence undermines the sustainable development of nations and the foundations of democratic institutions. Because of its complexity, the issue can be overwhelming. Several decades ago, Martin Luther King, who received the Nobel Peace Prize in 1964, asserted on violence: "We have flown the air like birds and swum the sea like fishes, but we have yet to learn the simple act of walking the earth like brothers."

In this special issue of the Revista Panamericana de Salud Pública/Pan American Journal of Public Health, researchers from the Region of the Americas and from Spain look at physical violence between persons and contribute to understanding it from the perspective of the behavioral sciences. Their work describes the results of the Multicenter Study: Cultural Norms and Attitudes Toward Violence in Selected Cities of Latin America and Spain (the ACTIVA project) and enumerates proposals and initiatives to address the problem. The ACTIVA project, an inter-American collaborative initiative between the Pan American Health Organization (PAHO) and research centers in seven countries, was carried out in accordance with a new plan for international partnership in public health research. In one of the articles in this issue, R. de los Ríos describes the project's basic principles. PAHO identified institutions and researchers, established coordination among them, and contributed the technical cooperation and financing needed to develop the study protocol, field test a questionnaire, review and compile databases, and disseminate the results. The researchers secured the funding for data collection and analysis in their individual cities as contributions from different local institutions, which made it possible to launch and carry out such a large undertaking. Seven cities in Latin America and one city in Spain participated in the project: Rio de Janeiro, Brazil; Salvador, Bahia, Brazil; Santiago, Chile; Cali, Colombia; San Jose, Costa Rica; San Salvador, El Salvador; Madrid, Spain; and Caracas, Venezuela. The researchers, who were professionals affiliated with academic and research institutions, were experienced in population studies and public health and had diverse training and backgrounds in social research. Sociologists, social psychologists, anthropologists, demographers, public health professionals, statisticians, and political scientists were all among the group of researchers who headed the project in each country. Thanks to the assistance of academic and research institutions, it was possible to secure both the researchers' time and the technical and logistical support needed to carry out the project.

The ACTIVA project strengthened exchanges between academic centers in Latin America and in the United States of America. Vital contributions came from the Health Promotion Center of the School of Public Health at the University of Texas in Houston and from the Department of Health Promotion and Behavior at the University of Georgia. Those two institutions obtained the assistance of subject specialists and brought together professors and researchers from their universities to design the study and to analyze and publish the results. Discussion of the results of the ACTIVA project was greatly facilitated by the Rockefeller Center for Latin American Studies at Harvard University, in Boston, which offered an opportunity for exchange by organizing and sponsor- 
ing a seminar that assembled project researchers and prominent experts and researchers, as well as persons directing violence prevention programs in the United States.

The ACTIVA project assessed the relationship between normative, attitudinal, and environmental factors on the one hand, and the prevalence of violence against the mate, children, and people outside the family on the other. Adopting a common approach and methodology made it possible to establish comparisons and to examine the regularities observed in different sociocultural urban contexts, as well as to identify important differences in the rates of violent deaths from intentional causes.

This special issue contains eight original articles based on data collected through the ACTIVA project. The first article, by M. Fournier et al., describes the conceptual foundations of the project and its methodology. Three other articles present studies comparing participating cities. One of them, by P. Orpinas, analyzes the personal, behavioral, and demographic factors associated with violence against family members and persons outside the family; another article, by F. Moreno Martín, looks at domestic violence; a third article, by J. M. Cruz, addresses the problem of victimization. Four articles describe specific aspects of violence in individual cities: Salvador, Bahia, Brazil (by C. V. Noronha et al.); San Salvador, El Salvador (by J. M. Cruz); Santiago, Chile (by E. Oviedo S. and A. Rodríguez); and Madrid, Spain (by F. Moreno Martín). Future issues of this or other scientific journals will publish additional studies drawing comparisons among cities included in the study, as well as individual reports on Rio de Janeiro, Brazil; Cali, Colombia; and Caracas, Venezuela. This issue also includes several appendices with the central constructs, dimensions, and sociodemographic variables, by source; frequency of responses among men and women; the questionnaire employed, and the sampling design in each city. All this may be valuable for other studies attempting to replicate the results or to explore them in greater depth.

The ACTIVA project is a step forward in better understanding the problem of violence in the Region of the Americas, particularly the role that cultural factors and socially learned behaviors play in shaping individual conduct. The project is not an exhaustive study of violence, which would be impossible. For instance, sexual violence, violence in schools and the workplace, and institutional violence were not addressed. Therefore, it is important to use these results in conjunction with those of other comparative studies in the Region, such as a recent study conducted by the Inter-American Development Bank, which assessed the economic impact of violence in six countries of Latin America. It is also important to compare the results of this study with ones from other studies carried out in the United States and in some countries of Latin America, particularly research dealing with violence against women and children, with urban crime and youth violence, and with violence and investment in social capital. Some of the studies have been sponsored by international organizations, such as PAHO, the Organization of American States, the United Nations Children's Fund, and the World Bank.

The issue of violence cannot be viewed exclusively on the personal level, that is, as a problem of individual behavior and attitudes. Policies that promote social equity, fundamental civil and human rights, access to education and health care, and opportunities for employment and dignified work are fundamental to maintaining and developing democratic and peaceful forms of social coexistence. Rigoberta Menchu, the Guatemalan woman who received the Nobel Peace Prize in 1992, emphasizes this point when she says that peace is based on the absence of conditions that lead to war and conflict, such as intolerance, disrespect, and, above all, hunger and a lack of dignity. Violence and its various expressions are rooted in the attributes of societies, not only the characteristics of individuals. The article by A. Pellegrini presents this controversy and the challenges it poses for research on violence as a public health problem, such 
as the adoption of new paradigms that go beyond the risk factor approach and beyond reducing and treating risk factors as if they were diseases.

The ACTIVA project sheds light on the personal factors that influence the development of violent behaviors, as well as the impact certain social norms have on people's individual behaviors. The results of this project can have important consequences for violence prevention policies. First, the studies described in this special issue clearly show that attitudes in support of violence and the inability to solve problems peacefully relate to specific violent actions against family members and others. As a result, interventions to change violent behaviors and teach people to handle conflicts should be an important part of programs to foster democratic practices, the exercise of citizenship, and tolerance and respect for differences, as well as of all those programs that seek to develop a code of civil ethics and a culture promoting peace.

Second, the ACTIVA studies found that having a firearm or wanting to have one was associated both with aggressive behaviors and with being a victim of aggression. There is also strong evidence that owning a gun increases the risk of being victimized. These findings, however, should be interpreted in light of the context so as to avoid drawing simplistic conclusions. In the United States, for example, where firearms control is lax, these weapons are involved in the majority of homicides. Establishing or intensifying gun-control measures should be a priority in the countries of the Region. However, this is a complex problem that should be viewed from a comprehensive perspective. Weapons control interventions and other forms of monitoring or alleviating violence should be part of more comprehensive programs to attenuate or eliminate unjust social gaps and differences.

Third, young men show the highest probability of both assaulting others and of being victims of criminal acts in public areas. Again, this phenomenon is not separate from the hardships suffered by some young people in terms of living conditions and work and educational opportunities; this confirms findings from other research, particularly in the United States. It also underscores the need to develop programs that increase social capital, expand the opportunities available to young people, and promote the organization and strengthening of social and community networks for male adolescents and youth.

Fourth, in some cities there is deep mistrust of the police and lawenforcement institutions, an attitude linked to the desire to have weapons for self-defense. Hostile responses from the police, particularly in poorer neighborhoods, have increased acts of violence in such areas and undermined the potential preventive effectiveness of the police in their relationship to the community. Interventions in support of law-enforcement call for specific studies and approaches in each city.

Fifth, excessive alcohol consumption appears to be a risk factor for victimization, as well as for using violence against the partner and against persons outside the family. Victimization and aggression both increase with more frequent drunkenness, again confirming results of other studies in the United States and some countries of Latin America. Policies to regulate access to and consumption of alcoholic beverages, especially among young people, could help reduce the problem of violence.

Sixth, the study shows a high prevalence of violence among couples. Although both men and women are victims and victimizers, other studies indicate the problem is much more serious when the woman is the victim. Most of the violence against women is rooted in economic, political, and cultural systems that reinforce male domination and control. In this study it was noted specifically that the lack of skills for conflict resolution and that attitudes supporting violence are associated with an increase of violence against the mate. Modifying these systems will help reduce violence against women. Finally, violence against children in the home is alarming. Furthermore, having been a victim of corporal punishment during childhood is a predictive factor for violence 
against the spouse or other partner. It is not surprising that various forms of violence are associated with each other, and the study confirms that. Those who behave violently with family members at home are also violent with people outside the family, in the social sphere.

The study reveals relationships and associations that make it possible to target action. Although admittedly not all results are conclusive or sufficient to explain the phenomenon of violence, this should not be an argument to immobilize the political, organizational, and technical willingness to implement programs based on a coordinated effort to prevent violence in the countries of the Region. The articles in the Current Topics section represent a small sampling of the interventions that are being conducted and evaluated in these countries. Monitoring systems, programs using the mass media, activities targeting young people, and attempts to prevent and control violence against women and children all share one characteristic: a concerted, united effort among different public, private, and civil institutions. These endeavors also lead us to an important conclusion from the public health perspective: problems are complex, and understanding them better calls for new paradigms and for creative approaches that go beyond merely controlling individual risk factors and instead seek a broader approach to disease prevention and health promotion based on the development of nations. 